\title{
The Effects of Chenodiol on Biliary Lipids and their Association with Gallstone Dissolution in the National Cooperative Gallstone Study (NCGS)
}

Scott M. Grundy, Shu-Ping Lan, John Lachin, the Steering Committee (Richard A. Baum, Robert L. Habig, Russell F. Hanson, Theodore Hersh, N. C. Hightower, Alan F. Hofmann, Elliott C. Lasser, Jay W. Marks, Hagop Mekhjian, Ronald Okun, Robert A. Schaefer, Leslie J. Schoenfield, Lawrence Shaw, Roger D. Soloway, Johnson L. Thistle, Fred P. Thomas, Malcolm P. Tyor), and the National Cooperative Gallstone Study Group University of Texas, Dallas, Texas 75235; George Washington University, Biostatics Center, Bethesda, Maryland 20014 (University of Maryland, Baltimore, Maryland 21201; Duke University, Durham, North Carolina 27710; University of Minnesota, Minneapolis, Minnesota 55455; Emory University, Atlanta, Georgia 30322; Scott and White Clinic, Temple, Texas 76501; University of California at San Diego, California 92103; Cedars-Sinai Medical Center and University of California at Los Angeles, California 90048: Ohio State University, Columbus, Ohio 43210; New York Hospital-Cornell Medical Center, New York 10021; University of Pennsylvania, Philadelphia, Pennsylvania 19104; Mayo Clinic, Rochester, Minnesota 55901)

bstract. The National Cooperative Gallstone Study was a double-masked trial conducted to determine the efficacy and safety of chenodeoxycholic acid (chenodiol) for dissolution of cholesterol gallstones. Patients with radiolucent gallstones were randomly allocated to either a high dose $(750 \mathrm{mg} / \mathrm{d}, n=305)$ or low dose $(375$ $\mathrm{mg} / \mathrm{d}, n=306)$ of chenodiol or placebo $(n=305)$ administered for 2 yr. Specimens of gallbladder bile were obtained for biliary lipid analysis on $50 \%$ of all white

This work was presented in part at the Annual Meeting of the American Gastroenterological Association, May 1982, in Chicago, IL.

Address all reprint requests to Dr. Kalser, National Institute of Arthritis, Diabetic and Digestive and Kidney Diseases, National Institutes of Health, Bethesda, MD 20205. Address all correspondence to Dr. Grundy, University of Texas, Dallas, TX 75235. The contents of this publication do not necessarily reflect the view or policies of the Department of Health and Human Services nor does mention of trade names, commercial products, or organizations imply endorsement by the U. S. Government.

Dr. Hanson is deceased.

Received for publication 21 March 1983 and in revised form 15 December 1983.

The Journal of Clinical Investigation, Inc.

Volume 73, April 1984, 1156-1166 patients at base line and after 3-mo therapy, on $45 \%$ at $12 \mathrm{mo}$, and on $36 \%$ at $24 \mathrm{mo}$. Among these specimens, $20 \%$ were inadequate for analysis. For analysis of data, available values during therapy were averaged up to time of dissolution, study exit, or study termination. In the high-dose group, percent chenodiol (molar percent of all bile acids) increased markedly and remained high during the $2 \mathrm{yr}$ of follow-up. Also, molar percent cholesterol decreased significantly and remained low during the $2 \mathrm{yr}$ of follow-up. In the low-dose group, percent chenodiol increased and remained significantly increased. Percent cholesterol saturation decreased at $3 \mathrm{mo}$, but at 24 mo it was not different from that in the placebo group, suggesting a physiological adaptation to the low dose by 2 yr.

$79 \%$ of patients on high dose had greater than $70 \%$ chenodiol. Among these, half showed unsaturated bile ( $<100 \%$ cholesterol saturation) while the remainder were supersaturated; in the former group with unsaturated bile, $23 \%$ had complete dissolution and $51 \%$ had partial $(>50 \%$ reduction in stone size) or complete dissolution. In contrast, those with over $70 \%$ chenodiol and supersaturated bile had only $5 \%$ complete dissolution. Thus, development of unsaturated bile was a major factor associated with 
Table I. Numbers of Patients with Adequate Bile Specimens Available for Analysis

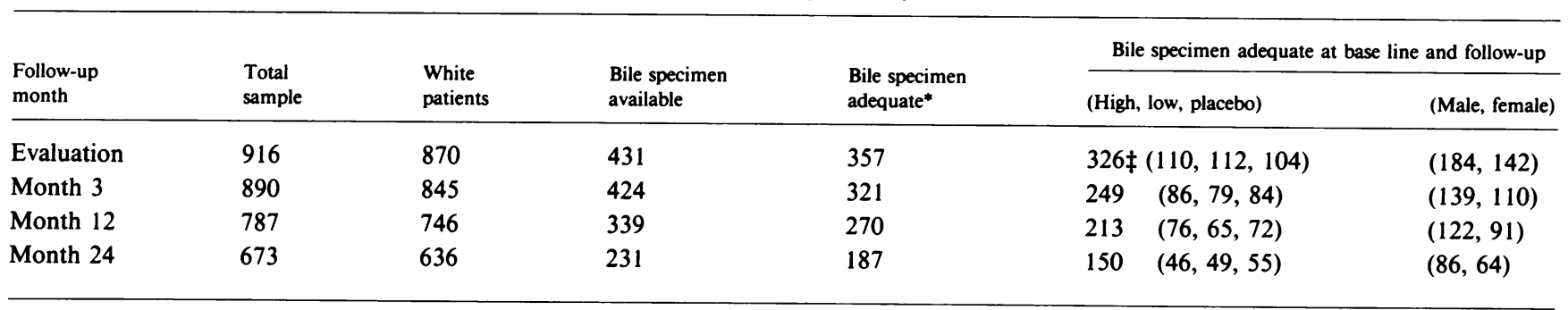

* A bile specimen is considered inadequate if: $(a)$ at least one of the lipids is unmeasurable, or $(b)$ bile acid is $<5 \mathrm{~mm}$, or $(c)$ bile acid is $<60$ molar percent, or $(d)$ contains $<0.6 \mathrm{~mm}$ of cholesterol. $¥$ Bile specimen available at base line and follow-up.

gallstone dissolution. The data also indicate that values for percent cholesterol saturation were a better predictor of gallstone dissolution than molar percent chenodiol, although a high percent chenodiol usually was required to obtain unsaturation.

\section{Introduction}

Several studies have shown that oral administration of the primary bile acid chenodiol (chenodeoxycholic acid) will dissolve cholesterol gallstones in some patients $(1-8)$. The probable mechanism of dissolution is to decrease the saturation of bile with respect to cholesterol (9). Recently, the results of a large clinical trial of chenodiol for dissolution of gallstones, the National Cooperative Gallstone Study (NCGS) ${ }^{1}$, were reported (10-15). This trial evaluated the efficacy and safety of two doses of chenodiol $(750$ and $375 \mathrm{mg} / \mathrm{d})$ in patients with radiolucent gallstones. Among 305 patients who were randomly assigned to receive the high dose for $2 \mathrm{yr}$, confirmed complete dissolution occurred in $14 \%$ of patients; partial (over $50 \%$ ) or complete dissolution were observed in $41 \%$. In 306 patients on the low dose, partial or complete dissolution occurred in $24 \%$, but complete dissolution in only $5 \%$. Of the remaining 305 patients who received placebo, only $10 \%$ and $1 \%$ had partial and complete dissolution, respectively. Dissolution was more common in women, thin patients, those with small or floating gallstones, and patients with high serum cholesterol within the normal range.

A substantial portion of the patients in this trial underwent duodenal intubation for collection of gallbladder bile at base line and during the study. Bile specimens were analyzed in a Central Bile Laboratory for biliary lipid composition and for individual bile acids. The purpose of this report is to summarize the results of these analyses; particular attention will be given to the effects of chenodiol administration on biliary lipid composition and dissolution of radiolucent gallstones.

\section{Methods}

Study design. The NCGS was a double-masked, controlled trial conducted to evaluate the efficacy and safety of randomly allocated high-dose (750 $\mathrm{mg} / \mathrm{d})$ or low-dose $(350 \mathrm{mg} / \mathrm{d})$ chenodiol or placebo administered for $2 \mathrm{yr}$ to 916 patients for dissolution of radiolucent gallstones in 10 clinical centers (10). Patients were of any race or sex; they were 21 to $79 \mathrm{yr}$ of age and weighed $<200 \%$ of ideal body weight. They had radiolucent gallstones discernible on oral cholecystogram (OCG) with no radiopacity on a plain film or minimal opacification ( $<3 \mathrm{~mm}$ nidus) on OCG. Only women unable to bear children were studied. Concurrent use of drugs suspected of interacting with chenodiol was proscribed. Further details of experimental design have been presented previously $(10,11)$.

Each patient underwent an initial screening for eligibility (the evaluation visit), and then, initiated a $30-d$ trial period to assess compliance. If eligible and consenting, the patient was randomized (the randomization visit), and thereafter, was evaluated at $1,2,3,6,9,12,16,20$, and 24 mo of follow-up. At each visit, a medical history was obtained and serum specimens collected for analysis locally and by a Central Serum Laboratory which performed assays of the serum aminotransferases (as serum glutamic-oxaloacetic transaminase [SGOT] and serum glutamicpyruvic transaminase [SGPT]) and lipids (cholesterol and triglycerides). A roentogenographic evaluation was conducted at the evaluation visit, and at months 9, 16, and 24 thereafter. Patients also were requested to undergo duodenal intubation for bile collection, but bile collection was not a requirement for enrollment in the study. Patients gave informed consent before intubation. Bile collection was carried out at either the evaluation visit usually within a month before randomization or at the randomization visit, and thereafter at months 3,12 , and 24 . The 3-mo sample followed $1 \mathrm{mo}$ at full dose of chenodiol, a period sufficient for observing pharmacological changes in saturation and lipid composition.

Study sample. A total of 916 patients were randomized into the NCGS; and bile specimens were collected at base line and during followup from consenting patients (Table I). Of these, $97 \%$ were still under study at month $3,86 \%$ at month 12 , and $73 \%$ at month 24 . Since $95 \%$ of the patients were white, and because of possible racial differences in biliary lipids, only white patients are included in this report; they accounted for $91 \%$ of all patients with bile samples at base line and $95 \%$ at each follow-up visit. Among the specimens, $20 \%$ were inadequate for inclusion in the analysis; the criteria for adequacy are described below under Bile Analysis. All total, 326 white patients (110 high-dose, 112 low-dose, and 104 placebo) had adequate specimens both at base line

1. Abbreviations used in this paper: GLC, gas-liquid chromatography; NCGS, National Cooperative Gallstone Study; OCG, oral cholecystogram; SGOT, serum glutamic-oxaloacetic transaminase; SGPT, serum glutamic-pyruvic transaminase. 
and any follow-up visit. These patients (37\% of total NCGS population) constitute the study sample for this paper. This percentage dropped to $29 \%$ at months 3 and 12 , and to $24 \%$ at month 24 .

Characteristics of the biliary lipid study sample were compared with all white patients of the NCGS. The few factors on which they differed are presented in Table II. The characteristics of the total study group were presented previously (10).

Laboratory methods. Bile samples were collected at the Treatment Centers, and the specimens were then stored in a refrigerator until shipment to the Central Bile Laboratory at the Veterans Administration Medical Center, San Diego, CA. In a previous report on pretreatment biliary lipid composition (12), detailed laboratory procedures were described for collection of bile samples, analysis of bile for lipids and individual bile acids, calculation of percentage saturation, collection and analysis of serum lipids and liver tests, and quality control of serum and bile analysis. All of these procedures were used in the present study.

When the bile acids contained $>2 \%$ lithocholic acid by gas-liquid chromatography (GLC), the fraction of lithocholic present as its sulfate was measured. The sulfated and unsulfated bile acids, along with internal radioactive recovery standards, were first separated on columns of Se-

Table II. Characteristics of All White Patients of the NCGS Study Population and White Patients with Adequate Bile Specimens

\begin{tabular}{|c|c|c|c|}
\hline & & Total* & Bile study \\
\hline \multicolumn{4}{|l|}{ Demography } \\
\hline & $\mathrm{N} \ddagger$ & 870 & 326 \\
\hline $\operatorname{Men}(\%)$ & & 46.8 & $56.4 \S$ \\
\hline \multirow[t]{2}{*}{ Ideal body weight (\%) $($ Mean \pm SEM) } & & 120.89 & $122.95 \S$ \\
\hline & & \pm 0.85 & \pm 1.32 \\
\hline \multicolumn{4}{|l|}{ Current smokers $\|$} \\
\hline Women (\%) & & 17.1 & $11.3 \S$ \\
\hline \multicolumn{4}{|l|}{ Roentogenographic characteristics } \\
\hline Floating stones (\%) & & 17.5 & $12.3 \S$ \\
\hline Single gallstone (\%) & & 29.4 & $34.1 \S$ \\
\hline \multirow{3}{*}{$\begin{array}{l}\text { Small countable stones } \\
\quad\left(<2.65 \mathrm{~cm}^{3}\right)(\%)\end{array}$} & & 34.3 & $29.8 \S$ \\
\hline & & & \\
\hline & $\mathbf{N} \ddagger$ & 516 & 193 \\
\hline \multirow[t]{2}{*}{ With $\leq 3$ countable stones } & & 24.2 & $19.2 \S$ \\
\hline & $\mathbf{N} \ddagger$ & 826 & 321 \\
\hline \multicolumn{4}{|l|}{ Biliary lipids } \\
\hline \multicolumn{4}{|l|}{ Cholesterol saturation } \\
\hline \multirow[t]{3}{*}{ (Hegardt and Dam) $(\% \pm$ SEM $)$} & & 144.59 & $139.17 \S$ \\
\hline & & \pm 2.37 & \pm 2.33 \\
\hline & $\mathbf{N} \ddagger$ & 513 & 326 \\
\hline
\end{tabular}

* Characteristics of total white population of NCGS.

$¥$ No. of patients in the total white population and those with adequate bile specimens for the characteristic shown. When a characteristic was not measurable on the whole population, the no. of patients included is given under the characteristic.

$\S$ Overall $P \leq 0.05$ comparing white patients with adequate bile specimens for base line and follow-up to the remaining patients without adequate bile specimens.

\| Smoked at least six cigarettes, two cigars, or two pipefuls of tobacco daily. phadex LD-20 (16). They were then subjected to solvolysis and quantitated by GLC using an SE-30 column. Losses during the procedure were corrected by using radioactive recovery standards of $\left[{ }^{14} \mathrm{C}\right]$ sulfolithocholyltaurine and $\left[{ }^{3} \mathrm{H}\right]$ lithocholylglycine.

The results for cholesterol, total bile acids, and phospholipids were calculated as millimoles per liter of bile and converted to molar percentages for each. The percent saturation was calculated according to the criteria of Hegardt and Dam (17), using the polynomial equations proposed by Thomas and Hofmann (18), assuming that bile contained $10 \%$ lipids by weight. Values for the percent saturation calculated by this method are essentially identical to those calculated using the critical tables of Carey (19), which are based on experimental data of Carey and Small (20), as described previously (12).

When samples were extremely dilute, they were considered inadequate for accurate analysis. Samples containing $<5 \mathrm{~mm}$ bile acids, or $0.6 \mathrm{~mm}$ cholesterol, or 60 molar percent bile acids were considered inadequate and have been excluded from the data base reported here.

Data management and statistical methods. All data management and statistical analyses were performed by using the Statistical Analysis System (SAS) (21). To confirm the accuracy of the GLC analysis for biliary bile acid composition, the 244 samples lying below the fifth or above the ninety-fifth percentile for percent of chenodiol, percent of cholic, or percent of deoxycholic acids were verified individually.

To compare characteristics between two groups, the chi-square statistic was used for proportions, and the $t$ statistic was employed for means. The method of weighted least squares analysis for contingency tables (22) was used to examine multidimensional categorical data and to test the significance of an effect (e.g., treatment), adjusting for the influence of covariates (e.g., sex). To examine the interrelationships among continuous variables, correlation coefficients were obtained. Multiple regression analyses were applied to investigate the proportion of variation in biliary lipids attributable to various combinations of patient characteristics. The coefficient of multiple determination $\left(\mathbf{R}^{2}\right)$ was used to represent the proportion of variation explained by the vari-

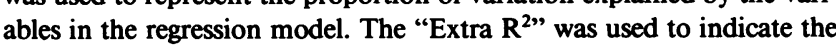
improvement of the prediction by the addition of a variable or set of variables after controlling for other variables (23). Logistic regression analysis (24) was used to generate predictive models for binary variables. Univariate analysis and trial models were used for the initial screening of possible predictors.

To depict changes in biliary lipids over time, the value of each component was adjusted by covariance for the base-line value and the mean adjusted values were plotted against time. The analysis of covariance adjusts the follow-up values as though each patient started out with the same base-line value (in fact, the group mean) based upon the regression equation of the follow-up values on the base-line values. A $t$ test or F test using the covariance adjusted values has greater statistical power to detect differences between groups than will a $t$ test or $F$ test based on simple changes from base line.

A $P$ value $<0.05$ was required for statistical significance, except for correlation coefficients which due to their multiplicity of testing were required to reach $P<0.01$. For pairwise comparisons among the three dose groups, a nominal $P<0.017$ was required for significance at the 0.05 significance level based on the Bonferoni inequality (25) to adjust for the effects of multiple testing.

\section{Results}

Values during follow-up. Mean levels for molar percent of each bile acid, adjusted for base-line values, are presented for men in Fig. 1 and women in Fig. 2. In both sexes, chenodiol feeding 


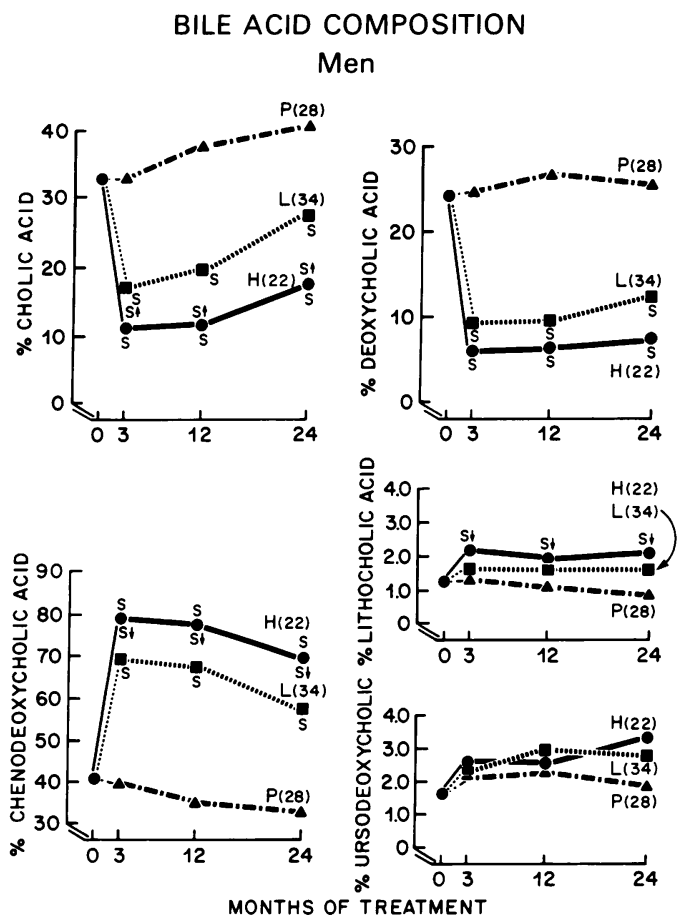

Figure 1. Bile acid composition: white males. Sample size 181, 137, 119 , and 84 , respectively, for base line; month 3,12 , and $24 . P$, placebo, $H$, high dose, $L$, low dose. S $\uparrow$ or $\downarrow: P<0.017$ high vs. low dose; S: $P \leq 0.017$ vs. placebo.

markedly increased percent chenodiol in both high-dose and low-dose groups; however, the increase was significantly greater in the high-dose than in the low-dose group. The increase in percent chenodiol was slightly greater in females than in males in both dosage groups. Although there appeared to be a slight drop in percent chenodiol at month 24 at both dose levels in men and women, the high percentages of chenodiol generally were maintained throughout the study. The percent chenodiol in the placebo group was unchanged.

Reciprocal changes were noted for both percentage of cholic acid and deoxycholic acid in all patients of both groups. Percentage of ursodeoxycholic acid appeared to be increased slightly during chenodiol feeding in men, whereas the differences between the groups were not significant; for women, the percentage of ursodeoxycholic acid was significantly increased in the treatment groups above that for placebo at months 3 and 12 (but not month 24). The high-dose group had a significant increase in percent lithocholic acid for men at all time points; for women, the increase was significant only at month 24 . On low dose, men did not have a significant increase in percent lithocholic acid, and women had a significant increase only at month 24 .

Mean levels for biliary lipids, adjusted for base-line values, are shown for men in Fig. 3 and for women in Fig. 4. For both sexes, percent cholesterol saturation fell markedly in the highdose group, and remained low throughout the study. The re- duction in percent saturation was paralleled closely by a fall in molar percent cholesterol.

For men in the low-dose group, percent saturation was reduced as much in the high-dose group over the first $3 \mathrm{mo}$, but at month 24 saturation rebounded back to levels approaching those of the placebo group. Women on low dose showed a somewhat less reduction in saturation in the first $3 \mathrm{mo}$, and the same rebound at month 24 also was noted. Thus, low-dose chenodiol appeared to lose its effectiveness in both sexes by month 24. As described for the major trial (10), among all patients, on average $92 \%$ of the intended dosage was actually received and these fluctuations in biliary lipids could not be explained by a progressive reduction in adherence. The same results were observed in analyses adjusting for fluctuations in body weight, and in analyses among the cohort of patients actually followed for 24 mo. Thus, the rebound in the low-dose group also was not influenced by weight change or the exit of patients from the study. For the placebo group, no consistent change in bile saturation was noted throughout the study in either men or women.

Correlations among biliary measures. Follow-up values for molar percent chenodiol at 12 and 24 mo were not correlated with base-line values in the high-dose group, but they were correlated highly with previous follow-up values (Table III). In the low-dose, 3-mo values were significantly correlated with base line, and the 12-mo value was correlated with the 3-mo percentage. However, a significant correlation with previous

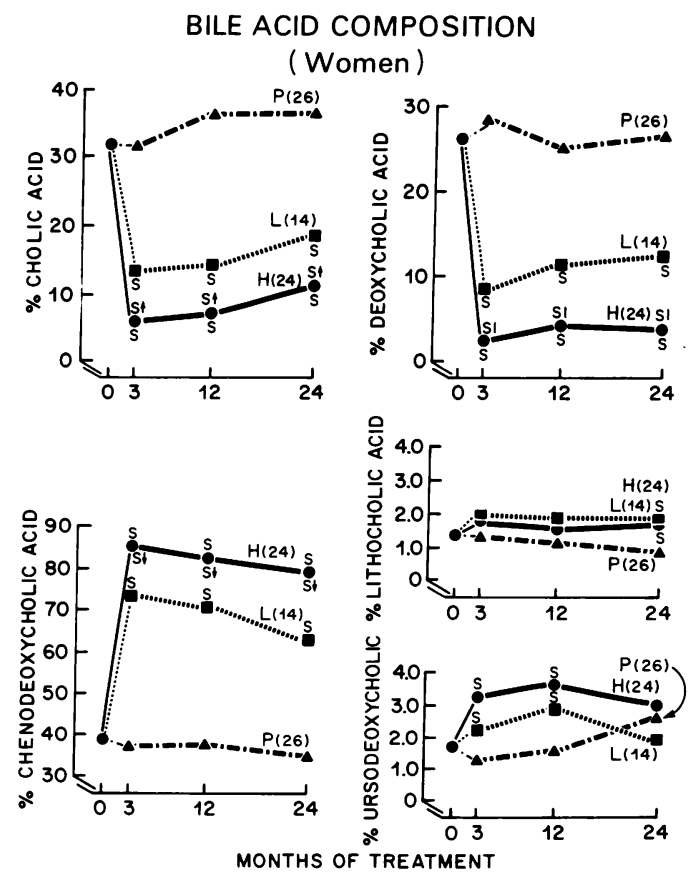

Figure 2. Bile acid composition: white females. Sample size 141, 108, 90 , and 64, respectively, for base line; month 3, 12, and 24. See Fig. 1 for symbols. 


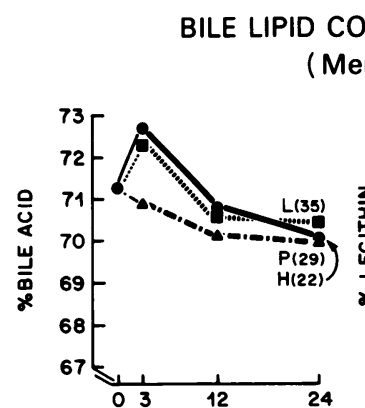

COMPOSITION
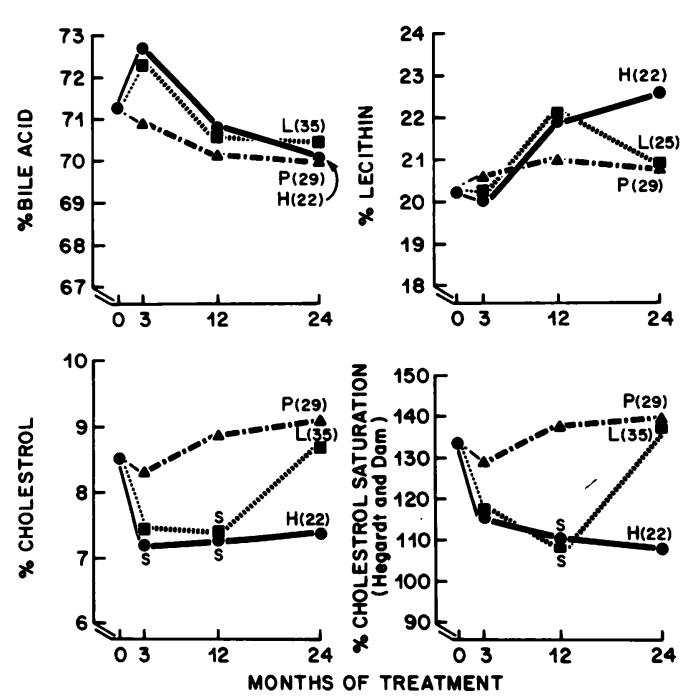

Figure 3. Bile lipid composition: white males. Sample size 181, 139, 119 , and 86, respectively, for base line; month 3, 12, and 24. See Fig. 1 for symbols.

values was lost at month 24 ; this latter may partially explain the rebound in percent saturation noted at month 24 in the low-dose group. For the placebo group, follow-up values generally were correlated with previous determinations. For percent saturation, correlations with previous values were less strong than for percent chenodiol.

\section{BILE LIPID COMPOSITION \\ (Women)}
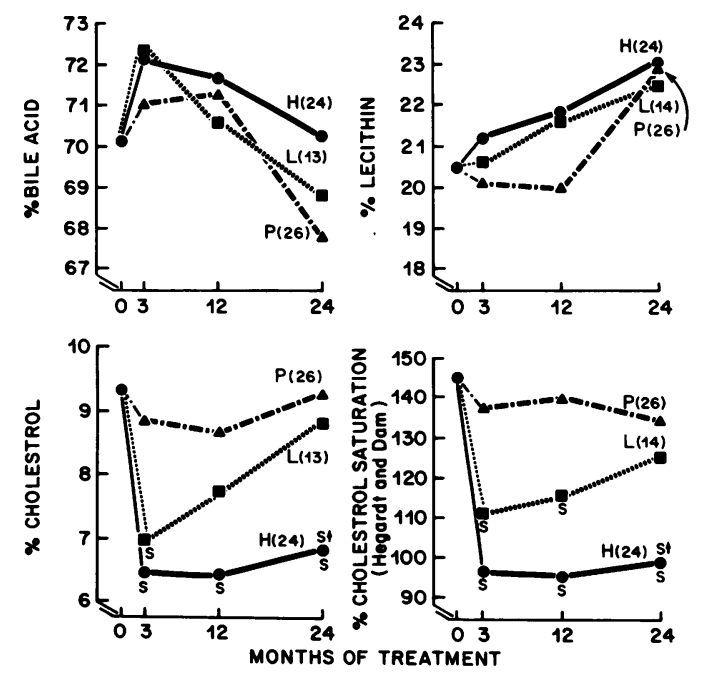

Figure 4. Bile lipid composition: white females. Sample size 141, 109,91 , and 63 , respectively, for base line; month 3,12 , and 24 . See Fig. 1 for symbols.
Table III. Correlation Matrices among Measurements at Base Line and at Follow-up Visits, for Chenodiol, and for Percent Cholesterol Saturation

\begin{tabular}{|c|c|c|c|}
\hline & Month 3 & Month 12 & Month 2 \\
\hline \multicolumn{4}{|l|}{ Chenodiol } \\
\hline \multicolumn{4}{|l|}{ High dose } \\
\hline Base line & -0.10 & 0.17 & 0.16 \\
\hline Month 3 & 1.00 & $0.51^{*}$ & $0.57^{*}$ \\
\hline Month 12 & & 1.00 & $0.75^{*}$ \\
\hline Month 24 & & & 1.00 \\
\hline \multicolumn{4}{|l|}{ Low dose } \\
\hline Base line & $0.30^{*}$ & 0.24 & 0.33 \\
\hline Month 3 & 1.00 & $0.65^{*}$ & 0.41 \\
\hline Month 12 & & 1.00 & 0.24 \\
\hline Month 24 & & & 1.00 \\
\hline \multicolumn{4}{|l|}{ Placebo } \\
\hline Base line & 0.28 & $0.51^{*}$ & $0.54^{*}$ \\
\hline Month 3 & 1.00 & $0.40^{*}$ & $0.46^{*}$ \\
\hline Month 12 & & 1.00 & $0.59^{*}$ \\
\hline Month 24 & & & 1.00 \\
\hline \multicolumn{4}{|c|}{ Percent cholesterol saturation } \\
\hline \multicolumn{4}{|l|}{ High dose } \\
\hline Base line & $0.34^{*}$ & 0.07 & 0.19 \\
\hline Month 3 & 1.00 & 0.26 & 0.13 \\
\hline Month 12 & & 1.00 & 0.33 \\
\hline Month 24 & & & 1.00 \\
\hline \multicolumn{4}{|l|}{ Low dose } \\
\hline Base line & $0.46^{*}$ & 0.09 & 0.01 \\
\hline Month 3 & 1.00 & $0.51^{*}$ & 0.32 \\
\hline Month 12 & & 1.00 & 0.07 \\
\hline Month 24 & & & 1.00 \\
\hline \multicolumn{4}{|l|}{ Placebo } \\
\hline Base line & 0.13 & $0.31^{*}$ & $0.39^{*}$ \\
\hline Month 3 & 1.00 & 0.27 & $0.50^{*}$ \\
\hline Month 12 & & 1.00 & 0.30 \\
\hline Month 24 & & & 1.00 \\
\hline
\end{tabular}

* Significant $P<0.01$.

The slopes of percent chenodiol in bile versus total body weight in the high-dose group are plotted separately for each visit in Fig. 5. During treatment, there was a significant inverse correlation between percent chenodiol and body weight at each visit. The reverse was noted for percent cholesterol saturation (Fig. 6).

The correlations between percent chenodiol and percent cholesterol saturation at each follow-up visit are presented in Table IV. There was a weak, but significant inverse correlation between percent chenodiol and percent saturation at base line ( $r=0.14, P<0.01$ ). For the high-dose group, during follow- 


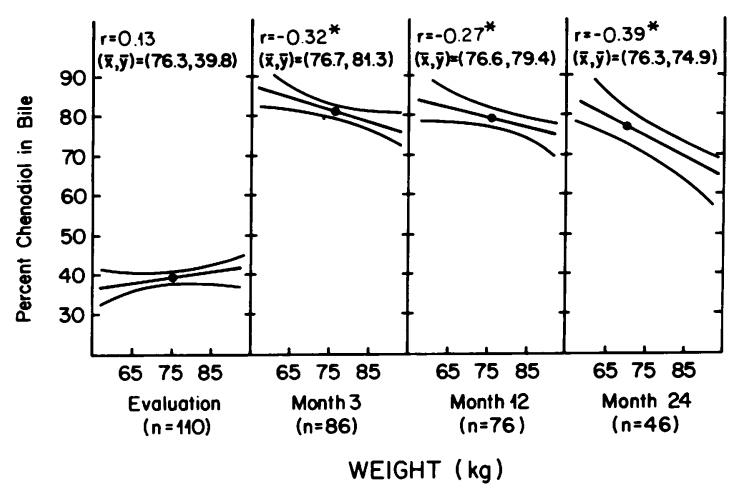

Figure 5. For high-dose group only. $r$ between body weight and percent chenodiol in bile, and regression ( \pm SE, $95 \%$ confidence band) of percent chenodiol in bile $(y)$ on body weight $(x) .(\bar{x}, \bar{y})$ represents the mean values for $x$ and $y .{ }^{*}, P \leq 0.05$.

up a consistent and significant inverse correlation was found at all visits. In the low-dose group, the relation was consistently inverse but not statistically significant throughout chenodiol therapy. In the placebo group, the inverse relationship held but was significant only at month 24 . Noteworthy was the consistency of inverse correlations at all visits.

When considering changes from base line (Table IV), the high-dose group showed a significant inverse correlation at month 3 between changes in percent chenodiol and percent cholesterol saturation $(r=0.130, P<0.01)$, but not at month 12 nor month 24 . The correlations for low-dose and placebo groups showed largely inverse relationships, but none were statistically significant.

Relation between biliary lipid composition and gallstone dissolution. Table $\mathrm{V}$ examines the rates of gallstone dissolution as a function of percent chenodiol (70\% vs. less) and biliary cholesterol unsaturation (100\% saturation vs. more) during follow-

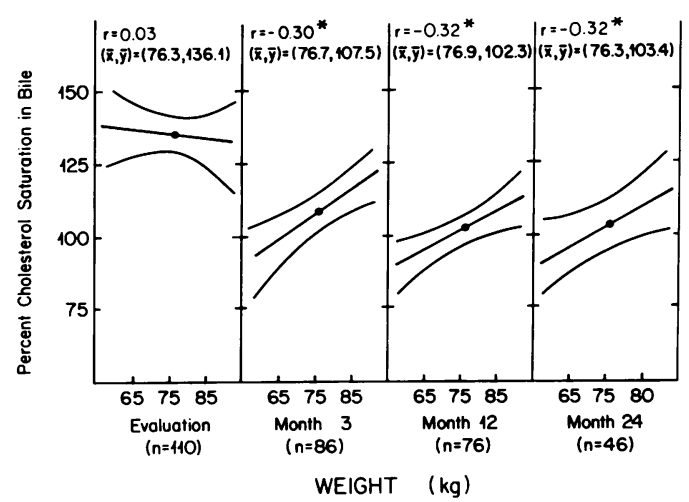

Figure 6. For high-dose group only. $r$ between body weight and percent cholesterol saturation, and regression $( \pm \mathrm{SE}, 95 \%$ confidence band) of percent cholesterol saturation $(y)$ on body weight $(x) .(\bar{x}, \bar{y})$ represents the mean values for $x$ and $y .^{*}, P \leq 0.05$. up; values were calculated as the mean of all follow-up values up to the time of dissolution or study exit or termination at month 24 . In the high-dose group, $79 \%$ of patients had an average chenodiol level of $>70$ molar percent. Among these, half showed unsaturation of bile; and of these, $23 \%$ had complete dissolution compared with $5 \%$ among those with saturated bile. All those with complete dissolution reached an average chenodiol level of $>70$ molar percent. Likewise, among those with $>70 \%$ chenodiol, $51 \%$ of patients with unsaturated bile experienced partial or complete dissolution compared with only $23 \%$ of those with saturated bile. For the 23 patients with $<70 \%$ chenodiol, most did not have unsaturated bile, none achieved complete dissolution, and only $16 \%$ experienced partial dissolution.

In the low-dose group, only $42 \%$ had $>70 \%$ chenodiol, and of these, $50 \%$ had unsaturated bile. Among the latter, none had complete dissolution, but $39 \%$ had partial dissolution. Among those with saturated bile, only $9 \%$ had complete dissolution, and only $17 \%$ showed partial or complete dissolution. Among those with $<70 \%$ chenodiol, $80 \%$ had saturated bile, and of these only one patient had complete dissolution. For the placebo group, none reached $70 \%$ chenodiol; most were saturated, and complete dissolution was not observed.

The above data were analyzed by weighted least squares methods for contingency tables to determine whether $>70 \%$ chenodiol or unsaturation were related to partial or complete dissolution. For the high-dose group, the effect of unsaturation was significant $(P<0.05)$ whereas percent chenodiol was not significant. Neither of these two effects were significant for the low-dose nor the placebo group.

Sample size in these analyses was limited (Table I). However, examination of the individual patient data revealed that complete dissolution did not occur with chenodiol below 65 molar percent; and of the 15 patients achieving complete dissolution, 13 had a percent chenodiol $>80 \%$. Also, complete dissolution did not occur in any patient with an average cholesterol saturation $>125 \%$, and two-thirds of those achieving complete dissolution had unsaturated bile.

Influence of weight. An association was found between weight and percent chenodiol and percent cholesterol saturation in the high-dose group, as shown in Figs. 5 and 6. For higher body weights, the percent chenodiol in bile declined; also, lighter body weights were associated with a greater degree of unsaturation. For these measurements, the sex effect as well as the interaction effect between sex and weight are not significant. Thus, the two sex groups are combined.

Predicting average percent cholesterol saturation during treatment with chenodiol. Multiple regression analyses were conducted to explore the possibility of predicting the average percent cholesterol saturation during follow-up. The variables that were of primary interest were weight, sex, average percent chenodiol, and percent cholesterol saturation at base line. Four sets of other variables were also considered: $(a)$ background variables: age, marital status, smoking status, and drinking status; (b) clinical variables: history of biliary pain, dyspepsia, and nausea within the past year; $(c)$ serum chemistries: serum GOT, 
Table IV. Correlation between Value and Change from Base Line of Percent Chenodiol with Value and Change from Base Line of Percent Cholesterol Saturation

\begin{tabular}{|c|c|c|c|c|c|}
\hline & At base line & At month 3 & At month 12 & At month 24 & Average during follow-up \\
\hline \multicolumn{6}{|c|}{ Correlations between values } \\
\hline High dose & -0.036 & $-0.335^{*}$ & $-0.398^{*}$ & $-0.40^{*}$ & $-0.377^{*}$ \\
\hline Low dose & -0.265 & -0.197 & -0.232 & -0.126 & $-0.248^{*}$ \\
\hline Placebo & -0.203 & -0.042 & -0.104 & -0.328 & -0.123 \\
\hline \multicolumn{6}{|c|}{ Correlations between changes from base line } \\
\hline High dose & & $-0.310^{*}$ & -0.148 & -0.039 & \\
\hline Low dose & & -0.154 & -0.142 & -0.197 & \\
\hline Placebo & & -0.028 & 0.033 & -0.146 & \\
\hline
\end{tabular}

* Significant $P<0.01$.

serum GPT, triglyceride, and cholesterol; $(d)$ radiologic variables: successful cholecystogram with single dose OCG, single stone, stone noncountability floating stones, volume of stones, number of stones, and whether the stones were small and countable.
Table VI presents the coefficients of multiple determination $\left(R^{2}\right)$ of the regression models within each treatment group. The contribution of a single variable or set of variables to the prediction of average percent saturation was evaluated by either

Table V. Gallstone Dissolution by Average Percent Chenodiol and Average Cholesterol Satuation

\begin{tabular}{|c|c|c|c|c|c|c|c|}
\hline \multirow[t]{2}{*}{ Average at follow-up } & \multicolumn{4}{|c|}{ Complete dissolution } & \multicolumn{3}{|c|}{ Partial or complete dissolution } \\
\hline & Total & $n$ & $\%$ & $P^{*}$ & $n$ & $\%$ & $P^{*}$ \\
\hline High dose & 110 & 12 & 10.91 & & 36 & 32.73 & \\
\hline Chenodiol > 70\% & 87 & 12 & 13.8 & & 32 & 36.8 & \\
\hline Unsaturated & 43 & 10 & 23.3 & $P<0.001$ & 22 & 51.2 & $P<0.05$ \\
\hline Saturated & 44 & 2 & 4.5 & & 10 & 22.7 & \\
\hline Chenodiol $<70 \%$ & 23 & 0 & - & & 4 & 17.4 & \\
\hline Unsaturated & 4 & 0 & - & NA & 1 & 25.0 & NS \\
\hline Saturated & 19 & 0 & - & & 3 & 15.8 & \\
\hline Low dose & 112 & 3 & 2.68 & & 25 & 22.32 & \\
\hline Chenodiol > 70\% & 47 & 2 & 4.26 & & 13 & 27.66 & \\
\hline Unsaturated & 23 & 0 & 0 & NS & 9 & 39.13 & NS \\
\hline Saturated & 24 & 2 & 8.70 & & 4 & 16.67 & \\
\hline Chenodiol $<70 \%$ & 65 & 1 & 1.54 & & 12 & 18.46 & \\
\hline Unsaturated & 13 & 0 & 0 & NS & 3 & 23.08 & NS \\
\hline Saturated & 52 & 1 & 7.69 & & 9 & 17.31 & \\
\hline Placebo & 103 & 0 & 0 & & 11 & 10.68 & \\
\hline Chenodiol $>70 \%$ & 0 & 0 & - & & - & & \\
\hline Unsaturated & 0 & 0 & - & NA & 0 & - & NA \\
\hline Saturated & 0 & 0 & - & & 0 & - & \\
\hline Chenodiol $<70 \%$ & 103 & 0 & - & & 11 & 10.68 & \\
\hline Unsaturated & 14 & 0 & - & NA & 2 & 14.29 & NS \\
\hline Saturated & 89 & 0 & - & & 9 & 10.11 & \\
\hline
\end{tabular}

Based on weighted least squares contingency table analysis for the high-dose group, the effect of "unsaturation" was statistically significant $(P<0.05)$, but the effect of "Chenodiol $>70 \%$ " was not. Neither of these two effects were statistically significant for the low-dose nor the placebo group. * $P$ of $\chi^{2}$ on $1 \mathrm{df}$, or where appropriate, of Fisher's exact test. 
entering that variable or the set in the model alone (model $\mathbf{R}^{2}$ ) or by considering its partial effects when added to the variables previously entered in the model (Extra $\mathbf{R}^{2}$ ). The regression model with all of the above variables explained $43 \%$ of the variation in the average percent saturation within the high-dose and the low-dose group and $26 \%$ within the placebo group.

Within the high-dose group, all the variables of primary interest were significant $(P<0.05)$ either entered individually or entered additively; together they accounted for $30 \%$ of the variation in average percent saturation. Background variables were significant when entered as the first set of variables to be considered, but were not significant after controlling for the primary variables. None of the clinical, laboratory, or radiology factors reached statistical significance. Within the low-dose group, the primary variables other than sex were significant, either when entered individually or additively, but sex was not. The primary variables accounted for only $13 \%$ of the variation in average percent cholesterol saturation. The significance in the background variables was primarily due to the finding that those not married $(n=13)$ showed lower average percent cholesterol saturation (mean \pm SE: $97.34 \pm 5.14$ for not married vs. $120.09 \pm 3.72$ for those married). The significance in the laboratory tests was due to a moderate but significant correlation $(r=0.27)$ between SGPT and average percent cholesterol saturation. None of the clinical or radiologic variables were significant. Within the placebo group, the only significant factor was the percent saturation at base line.

Estimating the probability of gallstone dissolution. Based on the complete sample of patients in the high-dose group $(n=305)$, logistic regression models were previously developed
(10) for the estimation of the probability of gallstone dissolution by using the most predictive variables among those obtained at base line. Among patients on high dose in this study ( $n$ $=110$ ), the following logistic models were obtained from the estimation of the probability of dissolution when average percent chenodiol and average percent cholesterol saturation during follow-up were added to these models. (The variable "Race" was not included because nonwhites were excluded from the bile study sample.) (a) Probability for complete dissolution $=\mathrm{e}^{\mathrm{y}} /(1$ $\left.+\mathrm{e}^{\mathrm{y}}\right)$ where $\mathrm{e}^{\mathrm{y}}$ is the antilog of $y$ and $y=-5.444-0.011$ $\times$ percent ideal body weight +0.925 (if cholesterol is $227 \mathrm{mg}$ / $\mathrm{dl}$ or above) +1.192 (if volume of countable stones is $<2.65$ $\left.\mathrm{cm}^{3}\right)+0.105 \times$ average follow-up percent chenodiol -0.054 $X$ average follow-up percent cholesterol saturation. (b) Probability for partial or complete dissolution $=\mathrm{e}^{\mathrm{y}} /\left(1+\mathrm{e}^{\mathrm{y}}\right)$ where $\mathrm{e}^{\mathrm{y}}$ is the antilog of $y$ and $y=0.888+1.170$ (if floating stones) +1.589 (if a woman) -0.364 (if stones not countable) +0.764 (if volume of countable stones is $<2.65 \mathrm{~cm}^{3}$ ) $-0.005 \times$ percent ideal body weight $+0.012 \times$ average follow-up percent chenodiol $-0.031 \times$ average follow-up percent cholesterol saturation.

The only variable that reached significance at the 0.05 level in the model for complete dissolution was average percent saturation; those in the model for partial or complete dissolution were sex and average percent saturation. The other variables had been significant in the analyses of the complete study sample, but did not reach significance levels in this study due to the reduction of sample size.

Based on the finding in the whole study that $13.5 \%$ of patients showed complete dissolution, the above models had an estimated sensitivity of $67 \%$ and a specificity of $77 \%$ in predicting complete

Table VI. Coefficients of Multiple Determination $\left(R^{2}\right)$ : Proportions of Variation in the Average Percent Cholesterol Saturation during Follow-up within Each Group Explained by Base-line Measurements

\begin{tabular}{|c|c|c|c|c|c|c|}
\hline & \multicolumn{2}{|c|}{ High dose } & \multicolumn{2}{|c|}{ Low dose } & \multicolumn{2}{|c|}{ Placebo } \\
\hline & Model $\mathbf{R}^{2 *}$ & Extra $R^{2 *}$ & Model $\mathbf{R}^{2 *}$ & Extra $\mathbf{R}^{2 *}$ & Model $\mathbf{R}^{2 *}$ & ${\text { Extra } R^{2 *}}^{2 *}$ \\
\hline & $\%$ & $\%$ & $\%$ & $\%$ & $\%$ & $\%$ \\
\hline All variables $\ddagger$ & - & $43.33 \S$ & - & $43.01 \S$ & - & 25.75 \\
\hline \multicolumn{7}{|l|}{ Primary variables } \\
\hline Weight & $11.68 \S$ & $11.18 \S$ & $4.91 \S$ & $5.28 \S$ & 0.59 & 0.80 \\
\hline Sex & $12.46 \S$ & $4.26 \S$ & 0.29 & .22 & 0.27 & 0.82 \\
\hline Average percent chenodiol & $14.20 \S$ & $6.63 \S$ & $6.16 \S$ & $3.86 \S$ & 1.51 & 1.07 \\
\hline Base-line percent saturation & $5.87 \S$ & $8.52 \S$ & $4.40 \S$ & $3.27 \S$ & $8.10 \S$ & $6.48 \S$ \\
\hline Background variables & $10.39 \S$ & 3.90 & 8.13 & $8.92 \S$ & 2.88 & 3.60 \\
\hline Clinical variables & 3.44 & 1.14 & 2.74 & 2.87 & 2.51 & 5.58 \\
\hline Laboratory measurements & 4.75 & 3.40 & $17.08 \S$ & $10.97 \S$ & 3.81 & 3.72 \\
\hline Radiology variables & 2.45 & 3.99 & 5.95 & 7.92 & 6.12 & 3.69 \\
\hline
\end{tabular}

* Model $\mathbf{R}^{2}$ is the proportion of variation in cholesterol saturation explanable by the set of variables alone. Extra $\mathbf{R}^{2}$ is the additional proportion explained by the set after controlling for proceeding sets. ‡ See text for specific variables included in the model. $§$ Significant at 0.05 level. 
dissolution. (Sensitivity is the proportion predicted to show dissolution among those with dissolution. Specificity is the proportion predicted not to show dissolution among those without dissolution.) Based on the finding that $40.8 \%$ of patients showed partial or complete dissolution, the above model had an estimated sensitivity of $69 \%$ and a specificity of $81 \%$.

Unsulfated lithocholic. For bile specimens with a lithocholic level $>2 \%$ ( $26 \%$ of specimens), the percents of sulfated and unsulfated lithocholic acids were determined. In these samples, the percent unsulfated lithocholic ranged from 6.5 to $93.3 \%$ with a mean \pm SE of $61.7 \pm 1.02 \%$. The effect of unsulfated lithocholic on liver function was examined by comparing the highest percent unsulfated lithocholic among those with vs. those without SGOT elevation, or SGPT elevation, or bilirubin elevation, or alkaline phosphatase elevation during the 24-mo study period. No association was found between percent unsulfated lithocholic acid and these liver function tests.

Adverse effects of chenodiol therapy. Table VII considers the relationship of percent chenodiol and percent cholesterol saturation with the principal adverse effects: SGPT elevations, serum cholesterol elevations, and diarrhea. Based on weighted least squares analysis, the percent chenodiol was significantly associated with SGPT elevations and diarrhea, but not with serum cholesterol elevations; it was also not significantly associated with SGOT elevations and rates of cholecystectomy (data not shown). Percent cholesterol saturation was not significantly associated with any of these outcomes.

\section{Discussion}

Determinants of bile saturation. This study (Figs. 1-4) showed that an intake of $750 \mathrm{mg} / \mathrm{d}$ chenodiol increased molar percent chenodiol in bile more than did $375 \mathrm{mg} / \mathrm{d}$. At both doses, the percent of chenodiol during treatment was greater in women than in men. Percent chenodiol declined slightly by 24 mo from the peak month 3 values in both sexes and at both doses. Increases in percent chenodiol generally were associated with reductions in percent cholesterol saturation. In men, the reduction in percent saturation was essentially the same in both doses in the first $12 \mathrm{mo}$; however, at month 24, the low-dose group showed a rebound in percent saturation to the placebo level. In contrast, the response in women to the high dose was consistently greater than to low dose; in fact, on the average, the bile was unsaturated in women on the high dose. This was the only group in which, on the average, bile was unsaturated on chenodiol therapy. In both sexes on high dose, no rebound in percent saturation occurred at 24 mo as was observed for the low dose.

There might be several reasons for the rebound in percent saturation observed in both sexes on the low dose, but analyses which accounted for adherence, weight change, and exit from the study before 24 mo produced similar results, i.e., all showed the rebound effect. Thus, the rebound most likely is a biological effect. This may be related to the decrease in percent chenodiol in bile as the study progressed, which may have been due to a loss of feedback control on bile acid synthesis towards the end of the 2-yr period.

Previous studies $(26,27)$ have suggested that, during chenodiol therapy, the percent chenodiol in bile is a major factor associated with a decrease in bile saturation. In this study, difference in the percent cholesterol saturation between high-dose and low-dose groups support these findings (Figs. 1-4). Furthermore, for both groups, there was an inverse correlation be-

Table VII. Adverse Effects by Average Percent Chenodiol and Average Cholesterol Saturation During Follow-up

\begin{tabular}{|c|c|c|c|c|c|c|c|}
\hline & \multirow[b]{2}{*}{ Total } & \multicolumn{2}{|c|}{ Serum cholesterol elevation } & \multicolumn{2}{|c|}{ SGPT elevation } & \multicolumn{2}{|l|}{ Diarrhea } \\
\hline & & $n$ & $\%$ & $n$ & $\%$ & $n$ & $\%$ \\
\hline Chenodiol $>70 \%$ & 134 & 106 & 79.1 & 35 & 26.1 & 90 & 67.2 \\
\hline Desaturated & 68 & 53 & 77.9 & 16 & 23.5 & 40 & 58.8 \\
\hline Saturated & 66 & 53 & 80.3 & 19 & 28.8 & 50 & 75.8 \\
\hline Chenodiol $<70 \%$ & 191 & 140 & 73.3 & 18 & 9.4 & 101 & 52.9 \\
\hline Desaturated & 31 & 27 & 87.1 & 2 & 6.5 & 20 & 64.5 \\
\hline \multirow[t]{3}{*}{ Saturated } & 160 & 113 & 70.6 & 16 & 10.0 & 81 & 50.6 \\
\hline & & \multicolumn{2}{|l|}{ Parameter } & \multicolumn{2}{|l|}{ Parameter } & \multicolumn{2}{|l|}{ Parameter } \\
\hline & & estimate & $\chi^{2}(d f=1)$ & estimate & $\chi^{2}(d f=1)$ & estimate & $\chi^{2}(d f=1)$ \\
\hline \multicolumn{8}{|l|}{ Test of Effect* } \\
\hline Intercept & & -1.25 & $66.79 \ddagger$ & 1.70 & $94.75 \ddagger$ & -0.40 & $10.02 \ddagger$ \\
\hline Chenodiol & & 0.89 & 0.36 & 0.65 & $14.67 \ddagger$ & 0.31 & $5.57 \ddagger$ \\
\hline Saturation & & -0.23 & 1.89 & 0.13 & 0.51 & 0.03 & 0.05 \\
\hline
\end{tabular}

* Based on weighted least squares contingency table analysis.

‡ Significant at 0.05 level. 
tween percent chenodiol and percent saturation; however, this was statistically significant only for the high-dose group (Table IV).

Weight was one of the factors, as observed in the major NCGS report (10), to influence the rate of dissolution with chenodiol feeding. The inverse relationship of weight to percent chenodiol (Fig. 5) and the direct relationship to percent cholesterol saturation (Fig. 6) probably can be explained by a positive correlation between body weight and synthesis (and biliary secretion) of cholesterol (28-31). Since more cholesterol is secreted in bile in obese patients, a given amount of oral chenodiol should produce less reduction in percent cholesterol saturation, as shown previously $(32,33)$. Unfortunately, because the NCGS was a fixed-dose trial, it was not possible to determine whether higher doses of chenodiol would override this effect of weight. Limited studies by other workers suggest that the obesity effect on percent saturation may be partially overcome by higher doses.

Determinants of gallstone dissolution. As reported in the major NCGS report (10), gallstone dissolution occurred significantly more frequently on the high dose than on low dose. Since dissolution was almost nonexistent in the placebo group, an appreciable increase in percent chenodiol probably was necessary to induce dissolution. In accord, the present study showed that in the high-dose group very few patients achieved partial dissolution and none had complete dissolution when percent chenodiol was $70 \%$ (Table V). The number of partial and complete dissolutions increased substantially when the level of chenodiol exceeded 70\%; and indeed, complete dissolution usually required $>80 \%$ chenodiol in bile. However, since the majority of patients receiving this high level of chenodiol did not show dissolution, the percentage of chenodiol alone was not sufficient to explain rates of dissolution. In none of the groups was a significant correlation obtained between percent chenodiol alone and dissolution rate.

A more important factor contributing to dissolution appears to be percent saturation. In fact, the greatest likelihood of dissolution was for those who achieved not only $70 \%$ chenodiol in bile but also unsaturated bile. Therefore, it would appear that the factor most responsible for gallstone dissolution was not the amount of chenodiol per se, but the presence of a low level of cholesterol saturation. From this study, it was not possible to determine whether desaturation of bile (i.e., saturation $<100 \%$ ) is required for gallstone dissolution. Many patients showed at least partial dissolution in the presence of saturated bile. However, since the analysis was based on a maximum of only three collections, it is unlikely that these could precisely reflect the average degree of saturation over a $2-y r$ period. Thus, the measurement of bile composition from a single specimen during chenodiol therapy probably would not be an adequate predictor for gallstone dissolution in individual patients.

Determinants of adverse events. In relation to the adverse events of chenodiol therapy described (10), an association was observed only between percent during follow-up ( $<70 \%$ vs. $>70 \%$ ) and the incidence of SGPT elevations and diarrhea. Percent unsulfated lithocholic acid, however, was not associated with signs of hepatotoxicity. Such an association was demonstrated by Marks et al. (34). In their study (34), however, percent unsulfated lithocholate was measured on all consenting patients, whereas in this paper, percent unsulfated lithocholate measurements were obtained only for those specimens with a percent total lithocholate $\geq 2 \%$.

Limitations of the study. This study is based on the 326 white patients who had adequate bile specimens both at base line and at any follow-up visit. This is approximately one-third of the total NCGS study sample. Also, there were a greater percent men and less floating stones in the present study sample than in the total NCGS sample. Therefore, the results of this study cannot necessarily be generalized to the total NCGS study. Another limitation of the study was the small number of samples taken on each patient. The composition of bile is known to vary from one day to another, and many more measurements might have provided a better prediction of gallstone dissolution.

\section{Acknowledgments}

This project was funded by the National Institute of Arthritis, Diabetic and Digestive and Kidney Diseases of the Department of Health and Human Services under contract number N01-AM-3-2216 and N01AM-0-2205.

\section{References}

1. Danzinger, R. G., A. F. Hofmann, L. J. Schonfield, and J. L. Thistle. 1972. Dissolution of cholesterol gallstones by chenodeoxycholic acid. N. Engl. J. Med. 286:1-8.

2. Bell, G. D., B. Whitney, and R. H. Dowling. 1972. Gallstone dissolution in man using chenodeoxycholic acid. Lancet. II:1213-1216.

3. Thistle, J. L., and A. F. Hofmann. 1973. Efficacy and safety of chenodeoxycholic acid for dissolving gallstones. N. Engl. J. Med. 289:655659.

4. Van Waes, L., M. De Weert, and M. Schurgers. 1975. Traitement de la lithiase biliare par l'acid chenique. Acta Gastro-enterol. Belg. 38:24-33.

5. Barbara, L., E. Roda, A. Roda, S. Casanova, D. Festi, C. Sami, G. Mazzella, and R. Aldini. 1977. Il trattamento medico della celcolosi biliare colesterolica nell' noma con acido chenodesossicolico. Minerva Med. 68:3355-3382.

6. Gerolami, A., H. Sarles, and R. Brette. 1977. Controlled trial of chenodeoxycholic therapy for radiolucent gallstones. A multicenter study. Digestion. 16:299-307.

7. Marks, J. W., J. H. Sherman, G. G. Bonorris, A. Chung, M. J. Coyne, and L. J. Schoenfield. 1978. Gallstone dissolution by chenodeoxycholic acid and phenobarbital. N. Engl. J. Med. 69:160-165.

8. Bateson, M. C., P. E. Ross, J. Murison, and I. A. D. Bouchier. 1978. Comparison of fixed doses of chenodeoxycholic acid for gallstone dissolution. Lancet. I:1112-1114.

9. Thistle, J. L., and L. J. Schoenfield. 1971. Induced alterations in composition of bile of persons having cholelithiasis. Gastroenterology. 61:488-496.

10. Schoenfield, L. J., and J. M. Lachin, The Steering Committee, and the National Cooperative Gallstone Study Group. 1981. Chenodiol (chenodeoxycholic acid) for dissolution of gallstones: the National Cooperative Gallstone Study. A controlled trial of efficacy and safety. Ann. Intern. Med. 95:257-282. 
11. Lachin, J. M., J. W. Marks, L. J. Schoenfield, the NCGS Protocol Committee, and the National Cooperative Gallstone Study Group. 1981. Design and methodological consideration in the National Cooperative Gallstone Study: a multi-center clinical trial. Controlled Clin. Trials. 2:177-229.

12. Hofmann, A. F., S. M. Grundy, J. M. Lachin, S.-P. Lan, R. A. Baum, R. F. Hanson, T. Hersh, N. C. Hightower, Jr., J. W. Marks, H. Mekhpian, R. A. Shaefer, R. D. Soloway, J. L. Thistle, F. B. Thomas, M. P. Tyor, and the NCGS Group. 1982. Pretreatment biliary lipid composition in white patients with radiolucent gallstones in the National Cooperative Gallstone Study. Gastroenterology. 83:738-752.

13. Albers, J. J., S. M. Grundy, P. A. Cleary, D. M. Small, J. M. Lachin, L. J. Schoenfield, and the NCGS Group. 1982. National Cooperative Gallstone Study: the effect of chenodeoxycholic acid on lipoproteins and apolipoproteins. Gastroenterology. 82:638-646.

14. Fisher, R. L., D. W. Anderson, J. L. Boyer, K. Ishak, G. Klatskin, J. M. Lachin, and M. J. Phillips, and the Steering Committee for the NCGS. 1982. A prospective morphologic evaluation of hepatic toxicity of chenodeoxycholic acid in patients with cholelithiasis: the National Cooperative Gallstone Study. Hepatology (Baltimore). 2:187-201.

15. Phillips, M. J., R. L. Fisher, D. W. Anderson, S.-P. Lan, J. M. Lachin, J. L. Boyer, and the Steering Committee for the NCGS Group. 1983. Ultrastructural evidence of intrahepatic cholestasis before and after chenodeoxycholic acid therapy in patients with cholelithiasis: the National Cooperative Gallstone Study. Hepatology (Baltimore). 3:209220.

16. Makino, I., H. Hashimoto, K. Schinozaki, K. Yoshino, and S. Nakagaua. 1975. Sulfated and nonsulfated bile acids in urine, serum, and bile of patients with hepatobiliary disease. Gastroenterology. 68:545553.

17. Hegardt, F. G., and H. Dam. 1971. The solubility of cholesterol in aqueous solution of bile salts and lecithin. Z. Ernaehrungswiss. 10:223233.

18. Thomas, P. J., and A. F. Hofmann. 1973. A simple calculation of the lithogenic index: expressing biliary lipid composition on rectangular coordinates. Gastroenterology. 65:698-700.

19. Carey, M. C. 1978. Critical tables for calculating the cholesterol saturation of native bile. J. Lipid Res. 19:945-955.

20. Carey, M. C., and D. M. Small. 1978. The physical chemistry of cholesterol solubility in bile. Relationship to gallstone formation and dissolution in man. J. Clin. Invest. 61:998-1025.
21. SAS Institute. 1982. SAS User's Guide: Statistics. SAS Institute Inc., Cary, North Carolina.

22. Grizzle, J. E., C. F. Starmer, and G. G. Koch. 1969. Analysis of categorical data by linear models. Biometrics. 25:489-504.

23. Draper, N. R., and H. Smith. 1966. Applied regression analysis. John Wiley \& Son, Inc., New York. 234-240.

24. Cox, D. R. 1970. Analysis of binary data. Chapman \& Hall, Ltd., London.

25. Miller, R. 1966. Simultaneous Statistical Inference. New York: McGraw Hill, Inc., New York. 101.

26. Iser, J. H., G. M. Murphy, and R. H. Dowling. 1977. Speed of change in biliary lipids and bile acids with chenodeoxycholic acid-is intermittent therapy feasible? Gut. 18:7-15.

27. Thistle, J. L., A. F. Hofmann, P. Y. S. Yu, and B. Ott. 1977. Effect of varying doses of chenodeoxycholic acid on bile lipid and biliary bile acid composition in gallstone patients: a dose response study. Dig. Dis. Sci. 22:1-6.

28. Grundy, S. M., A. L. Metzger, and R. Adler. 1972. Mechanisms of lithogenic bile formation in American Indian women with cholesterol gallstones. J. Clin. Invest. 51:3026-3043.

29. Bennion, L. J., and S. M. Grundy. 1975. Effects of obesity and caloric intake on biliary lipid metabolism in man. J. Clin. Invest. 56:9961011.

30. Mabee, T. M., P. Meyer, L. DenBesten, and E. E. Mason. 1976. The mechanism of increased gallstone formation in obese human subjects. Surgery (St. Louis). 79:460-468.

31. Shaffer, E. A., and D. M. Small. 1977. Biliary lipid secretion in cholesterol gallstones disease. The effect of cholecystectomy and obesity. J. Clin. Invest. 59:828-840.

32. Iser, J. H., P. N. Maton, G. M. Murphy, and R. H. Dowling. 1978. Resistance of chenodeoxycholic acid (CDCA) treatment in obese patients with gallstones. Br. Med. J. 1:1509-1512.

33. Mok, H. Y. I., K. vonBergmann, J. R. Crouse, and S. M. Grundy. 1979. Biliary lipid metabolism in obesity: effects of bile and acid feeding before and during weight reduction. Gastroenterology. 76:556-567.

34. Marks, J. W., S. O. Sue, B. J. Pearlman, G. G. Bonorris, P. Varady, J. M. Lachin, and L. J. Schoenfield. 1981. Sulfation of lithocholate as a possible modifier of chenodeoxycholic acid-induced elevations of serum transaminase in patients with gallstones. J. Clin. Invest. 68:1190-1196. 